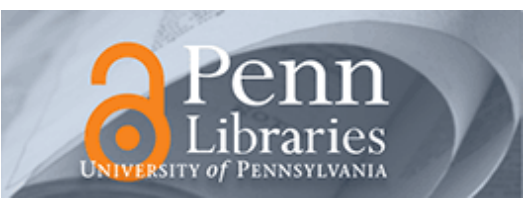

University of Pennsylvania

ScholarlyCommons

7-1-1997

\title{
Primary Adenocarcinoma of the Gland of the Nictitating Membrane in a Cat
}

\author{
András M. Komáromy \\ University of Pennsylvania, komaromy@vet.upenn.edu \\ David T. Ramsey \\ James A. Render \\ Phillip Clark
}

Follow this and additional works at: https://repository.upenn.edu/vet_papers

Part of the Ophthalmology Commons, and the Veterinary Medicine Commons

\section{Recommended Citation}

Komáromy, A. M., Ramsey, D. T., Render, J. A., \& Clark, P. (1997). Primary Adenocarcinoma of the Gland of the Nictitating Membrane in a Cat. Journal of the American Animal Hospital Association, 33 333-336.

Retrieved from https://repository.upenn.edu/vet_papers/46

PMID: 9204470

At the time of publication, author András Komáromy was affiliated with the Department of Small Animal Clinical Sciences, Michigan State University. Currently, he is a faculty member at the Vet Med school at the University of Pennsylvania.

Erratum

The wrong picture was used for Figure 3. The photomicrograph for Figure 3 on page 334 is of an impression smear of a metastatic lesion as described on page 335. The caption for Figure 3 is of an impression smear biopsy as described on page 333. The photomicrograph corresponding to this description was not published. (From JAAHA, 33(5), 369.)

This paper is posted at ScholarlyCommons. https://repository.upenn.edu/vet_papers/46

For more information, please contact repository@pobox.upenn.edu. 


\title{
Primary Adenocarcinoma of the Gland of the Nictitating Membrane in a Cat
}

\begin{abstract}
An 11-year-old, neutered, male domestic shorthair was presented with a five-month history of recurrent, unilateral, seromucoid discharge from the right eye. A verrucous mass extended from the posterior aspect of the nictitating membrane. Adenocarcinoma of the gland of the nictitating membrand (GNM) was diagnosed upon biopsy. The cat subsequently developed metastases to the lungs, pleura, mediastinum, liver, and kidneys and died six months after clinical signs first were observed. Little is known about the biological behavior of adenocarcinoma of the GNM in cats. This is the first report that describes the natural progression of this disease.

Disciplines

Medicine and Health Sciences | Ophthalmology | Veterinary Medicine

Comments

PMID: 9204470

At the time of publication, author András Komáromy was affiliated with the Department of Small Animal Clinical Sciences, Michigan State University. Currently, he is a faculty member at the Vet Med school at the University of Pennsylvania.

\section{Erratum}

The wrong picture was used for Figure 3. The photomicrograph for Figure 3 on page 334 is of an impression smear of a metastatic lesion as described on page 335. The caption for Figure 3 is of an impression smear biopsy as described on page 333. The photomicrograph corresponding to this description was not published. (From JAAHA, 33(5), 369.)
\end{abstract}




\section{Erratum}

The wrong picture was used for Figure 3 in "Primary Adenocarcinoma of the Gland of the Nictitating Membrane in a Cat" ( $\mathrm{J}$ Am Anim Hosp Assoc 1997;33:333-6). The photomicrograph for Figure 3 on page 334 is of an impression smear of a metastatic lesion as described on page 335. The caption for Figure 3 is of an impression smear of a tissue sample collected during the initial biopsy as described on page 333. The photomicrograph corresponding to this description was not published. We apologize for any problems this error may cause.

James A. Render, DVM, PhD,

Diplomate ACVP 


\title{
Primary Adenocarcinoma of the Gland of the Nictitating Membrane in a Cat
}

\begin{abstract}
An 11-year-old, neutered, male domestic shorthair was presented with a fivemonth history of recurrent, unilateral, seromucoid discharge from the right eye. $A$ verrucous mass extended from the posterior aspect of the rictitating membrane. Adenocarcinoma of the gland of the nictitating membrane (GNM) was diagnosed upon biopsy. The cat subsequently developed metastases to the lungs, pleura, mediastinum, liver, and kidneys and died six months after clinical signs first were observed. Little is known about the biological behavior of adenocarcinoma of the GNM in cats. This is the first report that describes the natural progression of this disease. J Am Anim Hosp Assoc 1997;33:333-6.
\end{abstract}

Andras M. Komaromy, Dr.med.vet.

David T. Ramsey, DVM, Diplomate ACVO

James A. Render, DVM, PhD, Diplomate ACVP

Phillip Clark, BVSc, MACVSc

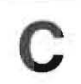

From the Department of Small Animal Clinical Sciences (Komaromy, Ramsey) and the Animal Health Diagnostic Laboratory (Render, Clark), Michigan State University, East Lansing, Michigan 48824-1314

Doctor Komaromy's current address is the Department of Small Animal Clinical Sciences, College of Veterinary Medicine, University of Florida, Gainesville, Florida 32610-0126.

\section{Introduction}

Adenocarcinoma of the gland of the nictitating membrane (GNM) has been reported in dogs and cats, ${ }^{1,2}$ but little is known about its biological behavior in cats. This report describes the clinical, gross, histological, and cylological features of a primary adenocarcinoma of the GNM in a cat.

\section{Case Report}

A $6.3-\mathrm{kg}, 11$-year-old, neutered, male domestic shorthair was presented to the Michigan State University Veterinary Teaching Hospital for evaluation of recurrent unilateral conjunctivitis involving the right eye. An ipsilateral, seromucoid ocular discharge had been present for five months. Previous trealment included oral antibiotic (i.e., amoxicillin and enrofloxacin) administration and topical administration of an antibiotic-corticosteroid ophthalmic preparation, ${ }^{a}$ but response to treatment was equivocal. One week prior to presentation, self-induced excoriation of the conjunctiva resulted in a hemorrhagic ocular discharge.

Abnormalities on physical examination were limited to the right eye. The nictitating membrane was protruded and hemorrhagic [Figure 1]. After instillation of a topical anesthetic, ${ }^{b}$ the bulbar aspect of the nictitating membrane was examined. A verrucous mass extended from the posterior aspect of the nictitating membrane and indented the adjacent cornea [Figure 2]. Other abnormal findings included bilateral nuclear sclerosis and a focal, hyperreflective area in the tapetal fundus of the left eye, medial to the optic nerve.

Results of a complete blood count (CBC) and a serum biochemical analysis were within the laboratory reference ranges. The cat was anesthetized, and several excisional biopsy samples were obtained from the posterior aspect of the mass. Impression smears of tissue samples were made and stained with a Romanowsky stain for cytological examination. The cellular component was composed of a monomorphic population of epithelial cells arranged primarily in clusters [Figure 3]. The nuclei were round-to-slightly oval with finely reticulated chromatin and inconspicuous nucleoli. Mitotic figures were infrequent. Cells had small amounts of moderately basophilic cytoplasm that was vacuolated irregularly. Cytological interpretation 


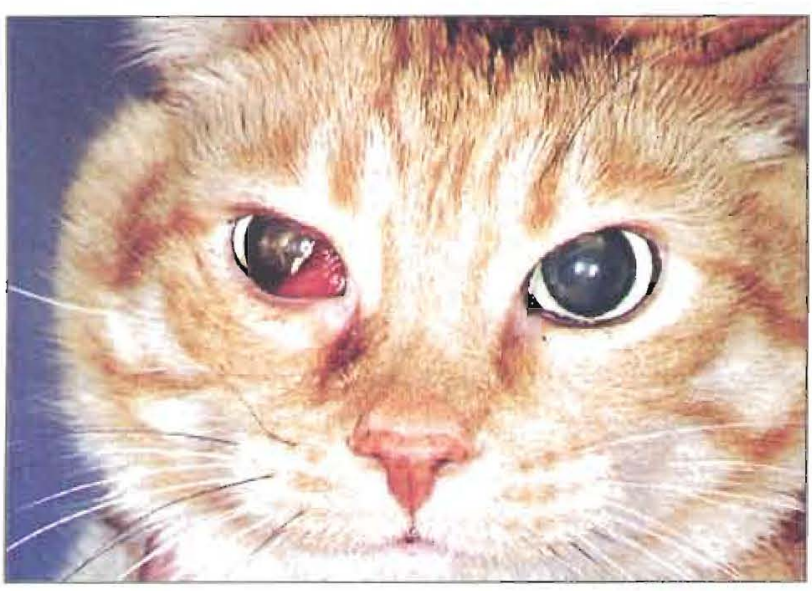

Figure 1-Photograph showing a cat with protrusion of the hemorrhaging, right nictitating membrane.

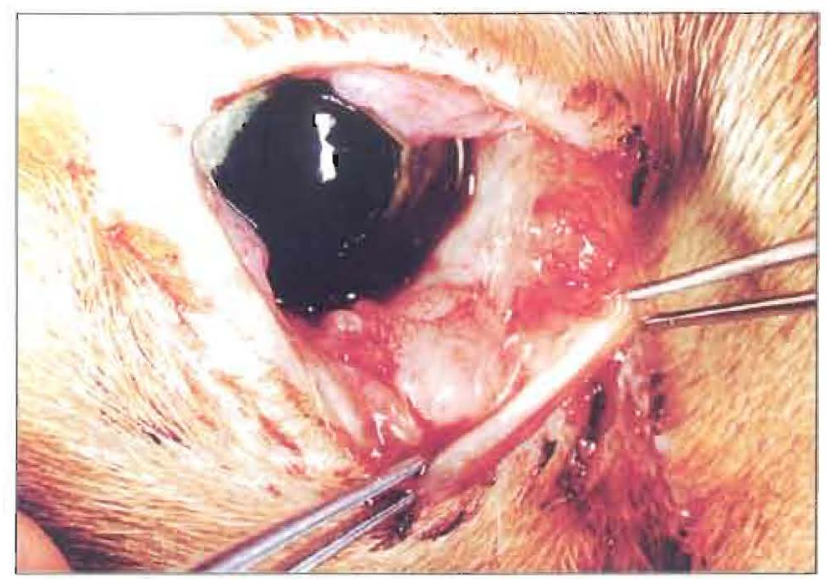

Figure 2-Photograph of the nictitating membrane from the cat in Figure 1. A verrucous mass on the posterior aspect of the nictitating membrane is evident.

was consistent with an epithelial neoplasm that had few criteria for malignancy.

The biopsies were submerged in neutral buffered $10 \%$ formalin, processed for paraffin embedding, and sectioned and stained for histological examination. Histological findings included a proliferation of $a b-$ normal glandular tissue on the palpebral and bulbar sides of the cartilage of the nictitating membrane This lissue, which effaced the normal glandular tissue, was composed of a uniform population of epithelial cells that had two morphological appearances [Figure 4]. The first appearance consisted of cuboidal-to-columnar epithelial cells arranged in acini and tubules. The lumens of glandular structures contained an eosinophilic secretory material. Epithelial cells had moderate amounts of cytoplasm and round, vesicular nuclei. The second appearance consisted of cells that were small, arranged in nests, and had scant cyloplasm, ovoidal nuclei, and dense chromatin. Mitotic figures (up to 10 per high-power field) were numerous. The surrounding connective tissue was ar-

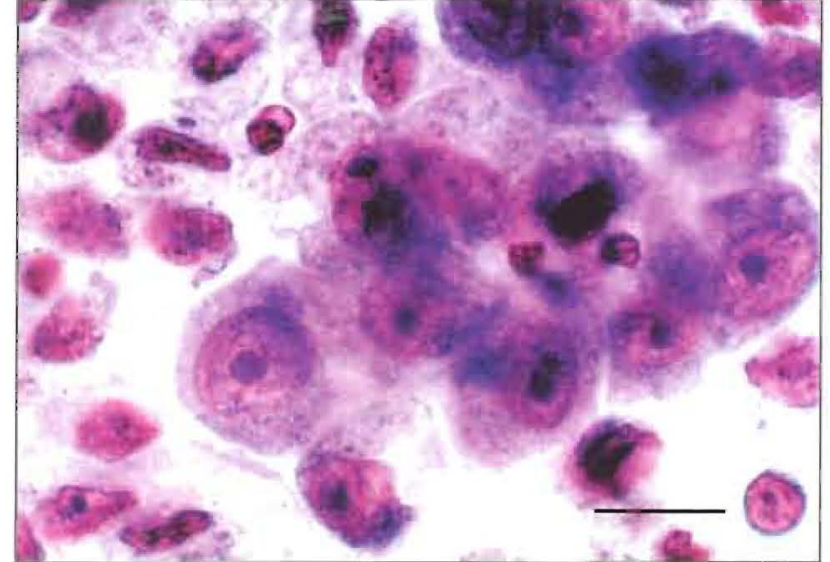

Figure 3-Photomicrograph of the impression smear obtained from the mass of the nictitating membrane. No malignant cytological criteria are evident (Romanowsky stain, 1,000X; bar=12 $\mu \mathrm{m})$.

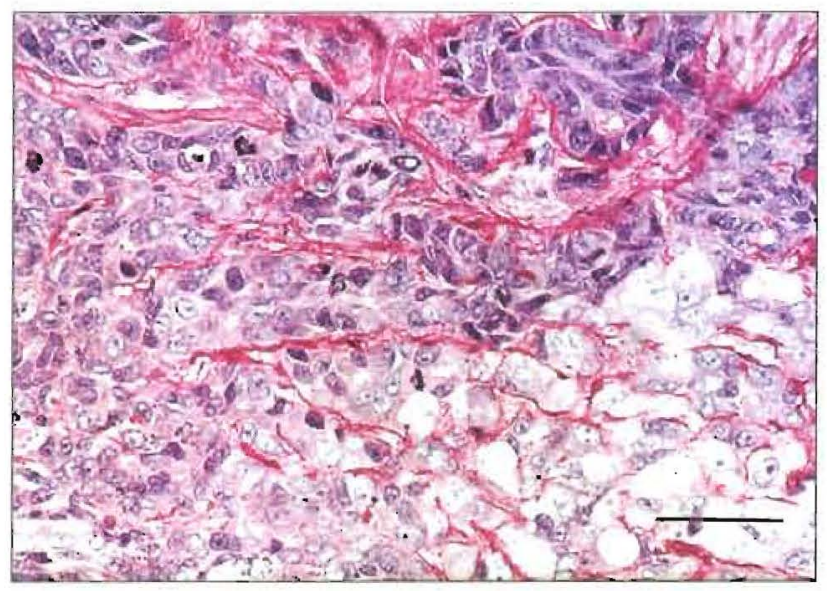

Figure 4-Photomicrograph of the biopsy taken from the mass in the right nictitating membrane showing sheets of neoplastic cells. Notice some cells resemble secretory epithelial cells with more cytoplasm and cytoplasm that is vacuolated. Other cells resemble basal epithelial cells and are polygonal to slightly fusiform with scant cytoplasm. Numerous mitotic figures are present (Periodic acid-Schiff stain, 40X; bar $=50 \mu \mathrm{m}$ ).

ranged loosely and had a mucinous-to-cartilaginous appearance. Neoplastic cells extended to the cut edge of the tissue, and areas of necrosis were present. The histological diagnosis was a lacrimal adenocarcinoma of the nictitating membrane. The owner declined further diagnostic tests or therapeutic procedures.

Four weeks after initial presentation, the cat was reevaluated. Body weight had decreased by $1 \mathrm{~kg}$. No difference in appearance of the mass was apparent during ophthalmic examination; however, exophthalmos of the right eye was evident.

Thoracic radiographs were made. Diffuse, indistinct, coalescing masses of the pulmonary parenchyma and rounding of the right costophrenic angle were evident [ligure 5]. Widening of the cranial mediastinum was suggestive of thoracic lymphadenopathy. Findings were supportive of pulmonary metastases 


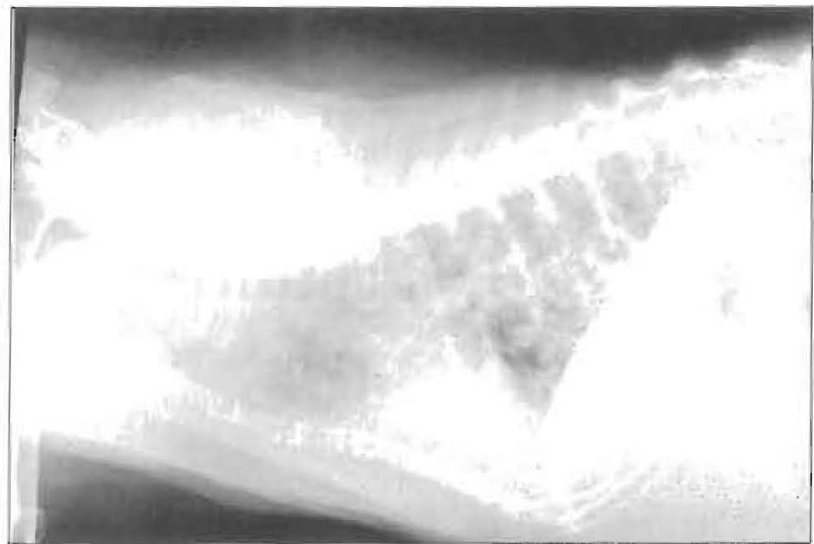

Figure 5-Diffuse, indistinct, coalescing masses in the pulmonary parenchyma are evident in this lateral thoracic radiograph of a domestic shorthair with adenocarcinoma of the gland of the nictitating membrane.

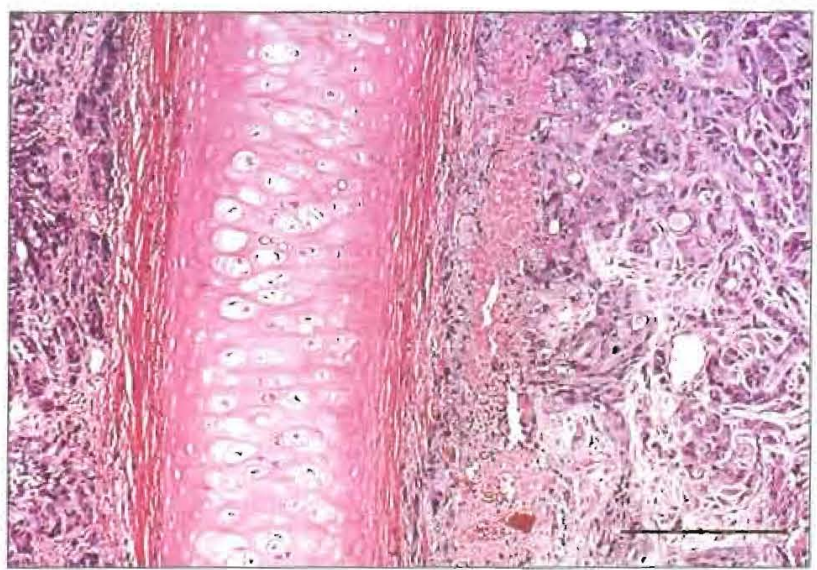

Figure 6-Photomicrograph of a section of the right nictitating membrane. Normal glandular architecture is lost completely along both sides of the cartilage. Architecture has been replaced by a uniform population of pleomorphic epithelial cells, primarily arranged in clusters (Hematoxylin and eosin stain, $20 \mathrm{x}$; bar $=200 \mu \mathrm{m}$ )

and mild pleural effusion; however, fungal disease could not be excluded. Serologic titers for Aspergillus sp., Blastomyces dermatitidis, Coccidoides immitis, and Histoplasma capsulatum were considered negative. The cat died three days later after sudden respiratory distress and seizure-like activity. The body was submitted for necropsy examination.

The entire right nictitating membrane was thickened and effaced by a mass that had an irregular surface and a mottled, white-red appearance. Histological findings included etfacement of the cartilage by a uniform population of pleomorphic epithelial cells arranged in sheets and well-demarcated aggregates of glandular structures [Figure 6]. Central areas of caseous necrosis were detected in some locations of the mass. The cells had a high nuclear-to-cytoplasmic ratio, a prominent nucleolus, and the cytoplasm was vacuolated which resembled that of secretory cells. Some cells with dense nuclear chromatin and

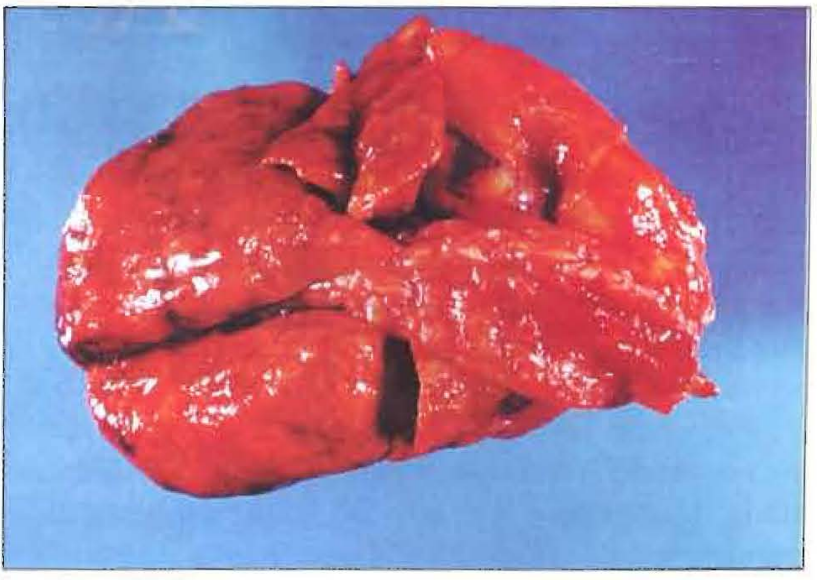

Figure 7-Photograph of the lungs containing numerous, coalescing, nonraised, metastatic, neoplastic nodules of variable sizes scattered throughout the entire pulmonary parenchyma.

scant cytoplasm resembled basilar cells. The mitotic index ranged from seven-to- 10 per high-power field. Neoplastic cells also infiltrated the connective tissue of the medial canthus and medial lower eyelid but did not involve the sclera. Neoplastic cellular aggregates were detected in blood vessels. The left eye and nictitating membrane were unremarkable.

Both lungs had di:seminated, multiple, white, firm, spherical, and irregular masses that coalesced and obliterated approximately $70 \%$ of the pulmonary parenchyma [Figure 7]. Areas of discrete necrosis and hemorrhage wert present within the masses. Several raised, white, pedunculated, spherical masses measuring approximately $3 \mathrm{~mm}$ in diameter were present in the parietal pleura on both sides of the thorax, ventrocaudal mediastinum, bifurcation of the trachea, hepatic parenchyma, and both renal parenchyma. The morphological appearance of cells and formation of necrotic centers in cellular aggregates were found consistently in all metastatic sites.

Impression smears made from presumed metastatic sites were dried and stained with a Romanowsky stain. Results of cytological analysis included a moderately high number of epithelial cells arranged in clusters within a proteinaceous background containing a moderate number of macrophages, few neutrophils, and erythrocytes. Individual cells were pleomorphic, anisokaryotic, and had moderately basophilic, finely granular cytoplasm. They had round-to-ovoid nuclei; coarse chromatin; a high nuclear-to-cytoplasmic ratio; and single, prominent nucleoli. Features were consistent with metastatic adenocarcinoma

\section{Discussion}

Disorders of the nictitating membrane occur infrequently in cats. Cystic disorders and prolapse in some feline breeds (e.g., Burmese) may be confused with neoplastic conditions. ${ }^{3,4}$ Neoplasms of the feline nic- 
titating membrane have been reported infrequently in the veterinary literature. ${ }^{2,5-7}$ Most reports are single case descriptions which support the assertion that these neoplasms occur infrequently. In one study, squamous cell carcinoma was reported to be the most common neoplasm involving the eyelid and the nictitating membrane, but the relative number of neoplasms affecting each anatomical location was not specified. ${ }^{7}$ In a recent retrospective study of feline orbital tumors, squamous cell carcinoma was considered the most common neoplasm. ${ }^{8}$ However, none of the neoplasms reported involved the nictitating membrane. Lymphoma also may infiltrate the nictitating membrane and its gland and result in piotrusion of the gland. ${ }^{4}$ Determining whether lymphoma primarily affects the nictitating membrane or is the result of extension of orbital disease may be difficult.

Neoplasms involving the nictitating membrane are reported more frequently in dogs than in cats. One retrospective study described the histological appearance and the clinical course of disease of adenocarcinoma in dogs.' Another retrospective study described neoplasms in the nictitating membrane of 47 dogs and five cats. ${ }^{2}$ Neoplasms in these five cats included adenocarcinoma $(n=2)$, squamous cell carcinoma $(n=2)$, and lymphosarcoma $(n=1)$.

Previous reports about adenocarcinoma of the nictitating membrane in cats have been limited to personal communication ${ }^{6}$ and to a brief description of two cases. ${ }^{2}$ The latter report described briefly the clinical course of the disease, histological appearance, and the biological behavior of adenocarcinoma of the GNM in two cats. The same report described 18 adenocarcinomas in dogs during the same time period of seven years. No sex predilection was observed in either species.

Similar characteristics are evident among adenocarcinoma of the GNM in $\operatorname{dog} \mathrm{s}^{1.2}$ and the cat in this report. The mean ages reported for affected dogs were $11.5^{1}$ and $11.3^{2}$ years and were similar to the age ( 11 years) of this cat. In dogs, the biological behavior of adenocarcinoma of the GNM is characterized by infiltrative growth, local recurrence, and lymph nodal and pulmonary metastases. ${ }^{1.2}$ In dogs and cats of previous reports ${ }^{1.2}$ and the cal in this report, the neoplasms were characterized histologically as well-differentiated acinar structures. However, mitotic figures were observed infrequently in most dogs ${ }^{1.2}$ while they were numerous in this cat. Time intervals to first recurrence of the neoplasm or death in dogs were two 1028 months, and five dogs did not have recurrence for 11 months to five years. ${ }^{1,2}$ In a four-year-old female cat. adenocarcinoma recurred three months after the initial excision. ${ }^{2}$ The age and gender in a second cat were not reported, but adenocarcinoma recurred seven months after initial excision, and the cat was euthanized subsequently. ${ }^{2}$ Comparisons of the biological behavior of adenocarcinoma of the GNM in dogs, 1.2 in two cats previously reported, ${ }^{2}$ and in the cat of this report must be interpreted with caution because the data is too limited to support statistically significant results. Nevertheless, in both species, this neoplasm has substantial malignant behavior and should be treated early and aggressively. In dogs, excision of the nictitating membrane has been recommended. 1.2 In the cat reported herein, natural progression of disease virtually was unimpeded and resulted in substantial morbidity and subsequent rapid mortality. The time from the first observed clinical sign until death was only six months. Rapid progression of the disease process in this cat suggests that surgical intervention at initial presentation may not have altered substantially the progression of the disease process.

\section{Conclusion}

The adenocarcinoma of the GNM in the cat reported herein had similarities to adenocarcinomas reported in dogs, including occurrence in an older animal, the initial nodular swelling, local infiltration, and pulmonary metastasis.' Natural progression of disease appears to be rapid and fatal. Immediate excision of the nictitating membrane is recommended when adenocarcinoma is detected.

a Gentocin Durafilm: Schering-Plough Animal Health. Kinciworth. NJ
b Alcuin: Alcon. Puerto Rico

\section{References}

I. Wilcock BP. Peiller RL Adenowarcmomat the gland of the third cyelid in seven Jogs J An Vet Med Assoc 1488:193-i $15+9-50$.

2. Schacfier 1:H. Pileghatar S, Goldon S. Knoedlsidur M. Mallgne

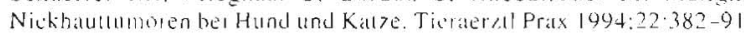

3. Koch SA Congenilal ophohal nic aboormalitics in the Burmese cal J Am Vet Med Axsol 1979:174:90-1

4. Nastsse MP Feline ophlhalmology. In: Gelatt KN. ed. Velerinary ophthalmolugy. 2nd ed. Philidelphia Lea \& Febiger. 1991:534

5. Buyuhmoci N. Fihrosarcomat of the nocutating membrane in a call J Am Vet Med Assoc 1975;167:934-5

6. Willams LW. Gelat KN. Gwin RM. Opbrhalmic neoplasms in the cat. J Am Anum Hosp Assoc 1981:17:999-1008.

7 MeLaughlın SA. Whitley RD, Gilger BC, Wright JC. Lindley DM Eyelid neoplasms in cats: a review of demographic data (1979-1989). J Am Anim Hosp Assoc 1993:29:63-7

8 Gilger BC. Mclitughlin SA. Whilley RD. Wright JC Orbital ncoplasms in cals 21 cilses (1974-1990) J Am Vet Med Assoc 1992:201:1083-6. 